\title{
Clinical Neonatal Brain MRI Segmentation Using Adaptive Nonparametric Data Models and Intensity-Based Markov Priors
}

\author{
Zhuang Song ${ }^{1}$, Suyash P. Awate ${ }^{1}$, Daniel J. Licht ${ }^{2}$, and James C. Gee ${ }^{1}$ \\ ${ }^{1}$ Departments of Radiology, University of Pennsylvania \\ ${ }^{2}$ Division of Neurology, The Children's Hospital of Philadelphia \\ Philadelphia, PA, USA 19104 \\ songz@seas . upenn. edu
}

\begin{abstract}
This paper presents a Bayesian framework for neonatal braintissue segmentation in clinical magnetic resonance (MR) images. This is a challenging task because of the low contrast-to-noise ratio and large variance in both tissue intensities and brain structures, as well as imaging artifacts and partial-volume effects in clinical neonatal scanning. We propose to incorporate a spatially adaptive likelihood model using a datadriven nonparametric statistical technique. The method initially learns an intensity-based prior, relying on the empirical Markov statistics from training data, using fuzzy nonlinear support vector machines (SVM). In an iterative scheme, the models adapt to spatial variations of image intensities via nonparametric density estimation. The method is effective even in the absence of anatomical atlas priors. The implementation, however, can naturally incorporate probabilistic atlas priors and Markovsmoothness priors to impose additional regularity on segmentation. The maximum-a-posteriori (MAP) segmentation is obtained within a graphcut framework. Cross validation on clinical neonatal brain-MR images demonstrates the efficacy of the proposed method, both qualitatively and quantitatively.
\end{abstract}

\section{Introduction}

Magnetic resonance imaging (MRI) has become an important tool for the clinical study of neonatal brain development and neurodevelopmental disorders [123. In neonatal brain MRIs, biological tissue properties, such as ongoing whitematter myelination and large water content, inherently limit inter-tissue intensity contrast-to-noise ratio (CNR), which is much lower than that of adult brain MRIs 124. Compared to adult brains, neonatal brains have larger variation in size, shape, and cortical-folding patterns. Furthermore, time constraints and subject motion may introduce significant imaging artifacts, noise, and field inhomogeneities. These problems are exacerbated in clinical neonatal brain imaging, which is typically optimized for visual inspection with low inter-slice resolution causing significant partial-volume effects.

N. Ayache, S. Ourselin, A. Maeder (Eds.): MICCAI 2007, Part I, LNCS 4791, pp. 883 890, 2007.

(C) Springer-Verlag Berlin Heidelberg 2007 


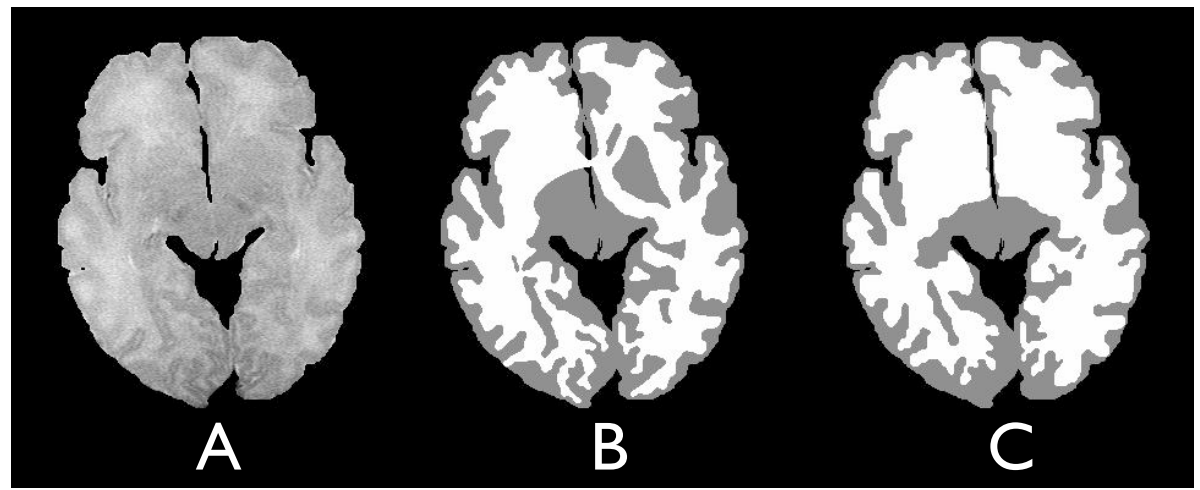

Fig. 1. An example slice of a neonatal brain T2-weighted axial MR image (A) and two manual segmentations of the same slice (B and C), showing significant disagreement

Several recent segmentation approaches 45.5 rely on probabilistic atlas priors that capture the spatial variability in the tissue structure of neonates. High quality atlases, however, are difficult to obtain, as there is often significant variability (especially near tissue boundaries and regions of myelination) in ground-truth manual segmentations from which the priors are derived (Figure 1). Because of low CNR and large variation in brain size and structures, incorporation of atlas priors also entails image registration which introduces another methodological challenge. These factors undermine the efficacy of the atlas-based approaches.

Another common element in segmentation approaches is the use of Gaussian probability density functions (PDFs) to model the tissue intensities. Such approaches have faced problems in adult brain MR tissue segmentation for producing anomalous behavior at low noise levels [6]. In tissue intensity histograms of neonatal brain MR images based on manual segmentations, the distributions are significantly different from Gaussians, which is consistent with the previously reported observation [4. Awate et al. modeled the Markov statistics of each tissue nonparametrically and have shown improvements over the Gaussian scheme in adult brain MR tissue segmentation [8].

We propose a Bayesian framework for neonatal brain tissue segmentation in clinical magnetic resonance (MR) images. It incorporates adaptive models using data-driven nonparametric statistical estimation schemes. Furthermore, the models adapt to spatial variations in image statistics that arise from variations in brain structure and tissue composition, in addition to imaging inhomogeneity. We present an effective strategy for constructing intensity-based Markov priors using fuzzy nonlinear support vector machines (SVMs) [10]. The proposed method can naturally incorporate atlas priors for additional regularity on the segmentation, although it is shown to be reasonably effective even without atlas priors. In this way, our method reduces the dependence on atlas priors, which helps to address the challenges in neonatal atlas construction. We compute the 
maximum-a-posteriori segmentation using graph cuts. Graph-cut techniques efficiently find global, or near global, optima for MRF-based energy function [1].

\section{Bayesian Segmentation with Markov Random Fields}

Consider an image $I$ defined on a set of voxels $\mathcal{T}$, the associated Markov neighborhood system $\mathcal{N}$, and an image of segmentation labels $L$. For voxel $t$, we denote the intensity by $I_{t}$, the segmentation label by $L_{t}$, the voxels in its Markov neighborhood by $\mathcal{N}_{t}$, and the intensities in the Markov neighborhood by $I_{\mathcal{N}_{t}}$. We consider the segmentation task as a Bayesian inference problem in a Markovrandom-field (MRF) framework. The optimal label assignment minimizes the Gibbs energy:

$$
E(L)=\sum_{t \in \mathcal{T}} V_{1}\left(L_{t}\right)+\sum_{t \in \mathcal{T}} \sum_{s \in \mathcal{N}_{t}} V_{2}\left(L_{t}, L_{s}\right)
$$

where $V_{1}(\cdot)$ and $V_{2}(\cdot)$ are the clique potentials associated with cliques of sizes 1 and 2, respectively, in a homogeneous MRF. We define

$$
V_{1}\left(L_{t}\right) \propto-\lambda_{1} \log P_{\mathrm{np}}\left(L_{t} \mid I_{t}\right)-\lambda_{2} \log P_{\mathrm{svm}}\left(L_{t} \mid I_{\mathcal{N}_{t}}\right)-\lambda_{3} \log P_{\mathrm{atlas}}\left(L_{t}\right),
$$

where $P_{\mathrm{np}}(\cdot)$ is the likelihood term obtained from a data-driven spatially-varying nonparametric model (described in Section 3), $P_{\mathrm{svm}}(\cdot)$ is the intensity-based prior learned using a fuzzy nonlinear SVM (described in Section 4), $P_{\text {atlas }}(\cdot)$ is the probabilistic atlas prior, and $\lambda_{1}, \lambda_{2}, \lambda_{3} \geq 0$ are the weight parameters, which are set empirically. Setting $\lambda_{3}=0$ ignores the atlas prior. The second term on the right side of the Gibbs energy function in (11) is defined as: $V_{2}\left(L_{t}, L_{s}\right)=1$ when $L_{t} \neq L_{s}$, otherwise $V_{2}\left(L_{t}, L_{s}\right)=0$. $V_{2}$ penalizes the differing label values in neighboring voxels, regularizing the segmentation. We minimize the energy in (11) using an efficient max-flow/min-cut algorithm [11, similar to that in [5].

\section{Adaptive, Nonparametric Tissue-Intensity Models}

Parametric modeling of tissue-intensity PDFs assumes that the forms of the PDFs are known. In many practical situations, especially those concerning neonatal MR images, simple parametric models may not accurately model the underlying data 4. The proposed method employs the Parzen-window nonparametric density estimation technique, which does not make strong assumptions about the forms of the PDFs, but rather infers the PDF structure from the input data. The Parzen-window probability estimate for each tissue-intensity $\operatorname{PDF} P\left(I_{t}\right)$, at voxel $t$, is:

$$
P_{n p}\left(I_{t} \mid L_{t}\right)=\frac{1}{\left|\mathcal{S}_{t}^{\prime}\right|} \sum_{i^{\prime} \in \mathcal{S}_{t}^{\prime}} G\left(I_{t} ; i^{\prime}, \sigma\right),
$$

where $\mathcal{S}_{t}^{\prime}$ is a random sample drawn from the tissue-intensity histogram for class $L_{t}$, and $G\left(I_{t} ; i^{\prime}, \sigma\right)$ is a Gaussian kernel with mean $i^{\prime}$ and variance $\sigma^{2}$. This allows us to compute the first term in (2), using Bayes rule,

$$
P_{n p}\left(L_{t} \mid I_{t}\right) \propto P_{n p}\left(I_{t} \mid L_{t}\right) / P_{n p}\left(I_{t}\right) .
$$


We have found that tissue-intensity statistics in virtually all MR images depict significant variability in different regions of the brain - this results from both biological properties and imaging artifacts. To adapt the model to such spatial variations, we use a local-sampling strategy. In this local-sampling framework, for each voxel $t$, we draw a unique random sample $\mathcal{S}_{t}^{\prime}$ from an isotropic Gaussian $\mathrm{PDF}$, defined on the image-coordinate space, with mean at the voxel $t$ and variance $\sigma_{\text {spatial }}^{2}$. Thus, the sample $\mathcal{S}_{t}^{\prime}$ is spatially biased and contains more voxels near the voxel $t$ being processed. Our experiments show that the method performs well for reasonable choices of $\sigma_{\text {spatial }}$ and encompasses more than a few hundred voxels. We choose $\sigma_{\text {spatial }}=6 \mathrm{~cm}$ and $\left|\mathcal{S}_{t}^{\prime}\right|=500$. Fixing $\sigma_{\text {spatial }}$ and $\left|\mathcal{S}^{\prime}\right|$, we chose a value for the kernel bandwidth $\sigma$ based on a standard penalizedmaximum-likelihood scheme 12 .

\section{Probabilistic Markov Intensity Priors Using SVMs}

This section describes the proposed strategy of learning intensity-based Markov priors using fuzzy nonlinear SVMs. The key idea underlying SVMs is to implicitly transform the data into a high-dimensional feature space and, subsequently, learn a linear classifier (hyperplane) in the transformed space [10. In this way, SVMs effectively learn complex nonlinear classifiers in the original feature space. Furthermore, finding linear classifiers reduces the optimization to a quadratic programming problem (involving inner product of feature vectors) that yields fast algorithms for finding the global optimum. SVMs avoid overfitting by computing an optimal classifier that maximizes the margin of separation between the classes. The classifier that separates the classes is described by only a fraction of training points in the feature space - namely, the support vectors, leading to efficient data representation and computation.

SVMs employ kernel functions $K(\cdot, \cdot)$ to implicitly transform the feature vectors (say $z_{i}, z_{j}$ ) to a higher-dimensional space $\mathcal{F}$ and evaluate their inner product, i.e. $\mathcal{F}\left(z_{i}\right) \cdot \mathcal{F}\left(z_{j}\right)=K\left(z_{i}, z_{j}\right)$. The proposed method effectively uses Gaussian kernels $K\left(z_{i}, z_{j}\right)=\exp \left(-\left\|z_{i}-z_{j}\right\|^{2} / \sigma_{\text {SVM }}^{2}\right)$. We chose $\sigma_{\text {SVM }}^{2}$ to be $10 \%$ of the image intensity range - the SVM output is fairly robust to small changes in this value.

Fuzziness is often required in segmentations to deal with uncertainty in decision making. This becomes more important for neonatal brain MR images because the myelination process makes it more difficult to differentiate between white matter and gray matter based on intensity alone. Fuzzy learning with SVMs, which allows misclassification on some training data, decreases the sensitivity to manual segmentation errors in a principled manner, and reduces chances of overfitting. As with typical fuzzy classifiers, the degree of fuzziness is specified by a free parameter, namely $C[13$. We chose $C=0.01$ to enforce a reasonable degree of fuzziness in the learned classifier. Figure 2(C) shows an example slice of the output of a fuzzy SVM. 
The SVM outputs numbers that indicate the distance of the test data point from the learned boundary that separates the classes. To calibrate the SVM outputs into probabilities, researchers have proposed, and rigorously analyzed, schemes akin to regularized maximum-likelihood approaches in statistics [14]. We employ a standard technique which fits a sigmoid function to the SVM outputs and converts them into prior probabilities [14.

\section{Experiments and Validation}

The difficulty of accurate manual segmentations on clinical neonatal brain MRI makes validation a challenging task. Figure 1 demonstrates the degree of disagreement between different human raters. In the light of these limitations, we propose to validate the segmentation of the proposed method, not only by quantitative comparison to the manual segmentations, but also by qualitative visual inspection. The challenges in automatic neonatal brain MRI segmentation often require multi-spectral image data 4415. Due to the poor image quality of the T1 weighted MRIs in our current dataset (based on the opinion of pediatric neurologists), we used only T2 weighted MRIs in the validation of our method. The proposed framework can handle multi-spectral images in a straightforward manner.

We performed cross validation (leave-one-out strategy) on clinical axial T2weighted images $(512 \times 384 \times 28$ with voxel size $0.35 \times 0.35 \times 3 \mathrm{~mm})$ scanned using a spin-echo pulse sequence on ten neonates - all term newborn infants with ages less than ten days. The proposed method considers myelinated and nonmyelinated white matter as a single tissue class - a postprocessing stage can help further differentiate the white matter, as in 4. Gray and white matter of all ten T2-weighted MR images were manually segmented by two experts. The skull and other brain tissues were removed using manual delineation. Probabilistic atlas priors were constructed by registering pre-segmented MRIs to a canonical atlas space. For this purpose, we used diffeomorphic flow-based registration and unbiased population-atlas construction [16]. We preprocessed the scanned MR images using standard techniques: (a) edge-preserving anisotropic smoothing, followed by (b) inhomogeneity correction using an information minimization method [17, and (c) adaptive histogram equalization to enhance local contrast [18. For effective SVM training, we ensured a reasonable match of the histograms of the preprocessed images. Because of the low inter-slice resolution typically found in clinical neonatal MR images, we chose the neighborhood $N(t)$, for each voxel $t$, as the $3 \times 3$ voxel neighborhood in the corresponding axial slice. We rescaled all images so that the intensities lie in the range of $[0,100]$. SVM training, using nine of the ten images, takes about two hours using SVMTorch [13.

Qualitatively, Figure 2 shows that the proposed method obtained an improved segmentation, in the marked areas, as compared to that using a Gaussian model based method. Note that the quantitative comparisons should be interpreted conservatively because of the challenges in ground-truth construction, as discussed previously. Figure 3(A) shows the Dice overlap metric for the ten neonates, for 


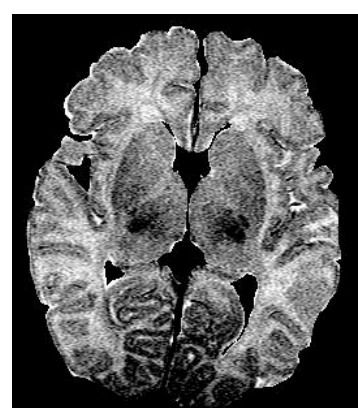

A

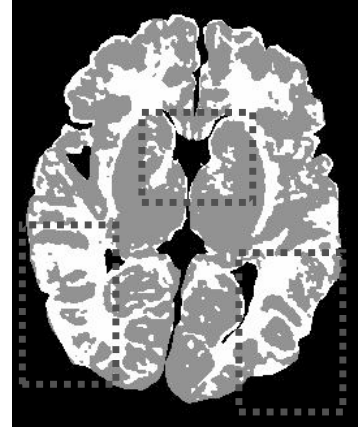

D

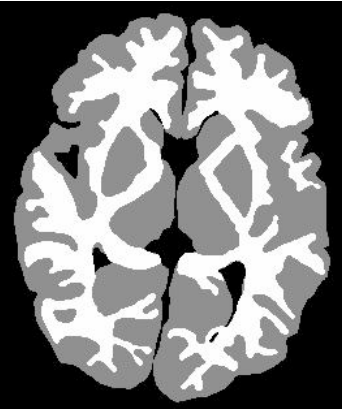

B

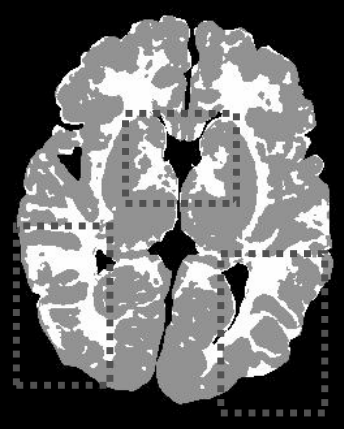

E

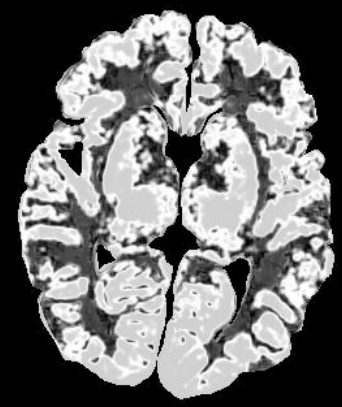

C

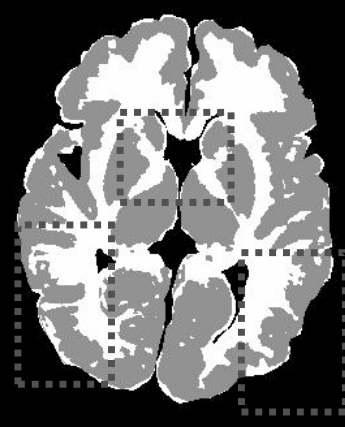

F

Fig. 2. Qualitative comparison of segmentation results. (A) original neonatal T2weighted MR image with only white matter and gray matter.(B) Manual segmentation by rater A. (C) Output of a fuzzy SVM for this image. Segmentations using: (D) conventional Gaussian tissue-intensity models, (E) adaptive nonparametric modeling using Markov-intensity-based priors learned by the fuzzy SVM $\left(\lambda_{1}=1, \lambda_{2}=1, \lambda_{3}=0\right)$, and (F) adaptive nonparametric modeling coupled with atlas priors $\left(\lambda_{1}=1, \lambda_{2}=1, \lambda_{3}=\right.$ $0.2)$.

both gray and white matter, to compare the automatic segmentations with the manual segmentations by rater A. As a reference, it also shows the performance of rater B based on the segmentations of rater A as the ground truth. In comparison to the results of the Gaussian model based method, the proposed method has higher Dice overlap values for gray matter. For the proposed method, using the atlas prior produces segmentations with slightly higher Dice overlap values. Figure 3(B) shows that the average brain-tissue volumes calculated from the segmentations of the proposed method lie between the volumes calculated from the two manual segmentations (with similar variances in all three cases). This indicates that the tissue-volume estimation by the proposed method can be as accurate as that provided by human experts. 


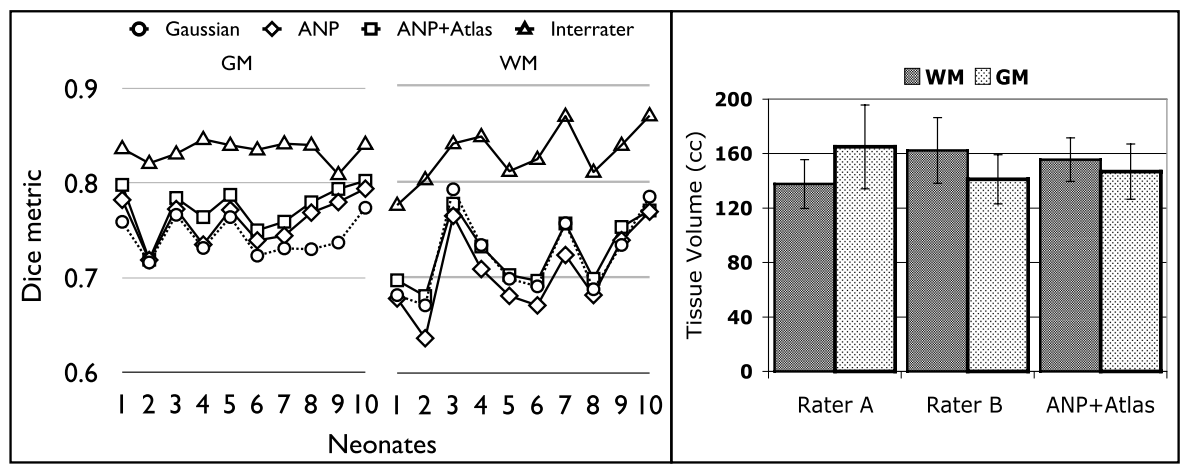

A

B

Fig. 3. Quantitative comparison of cross validation results: (A) Dice overlap metric of gray and white matters between the manual segmentation by rater $\mathrm{A}$ and four different segmentations based on: (i) the manual segmentation by rater B, (ii) Gaussian model with adaptive inhomogeneity correction, (iii) the proposed adaptive nonparametric (ANP) method, (iv) the proposed ANP method including the atlas prior (ANP+Atlas). (B) Volume of brain tissues given by the segmentations by rater $\mathrm{A}$, rater $\mathrm{B}$, and the proposed automatic method (ANP+Atlas).

\section{Conclusion}

We present a neonatal brain-MR segmentation method that produces a MAP segmentation using nonparametric likelihood models and intensity-based priors learnt by fuzzy nonlinear SVMs. The proposed nonparametric modeling adapts to the spatial variability in the intensity statistics that arises from variations in brain structure and image inhomogeneity. The proposed method produces reasonable segmentations even in the absence of atlas prior. Moreover, we find that intensity-based priors relying on empirical Markov statistics are more effective, than atlas-based priors, given high variability in manual segmentations and the challenges for registering neonatal images. Fuzzy learning with SVMs decreases the sensitivity to manual segmentation errors in a principled manner. Cross validation using ten neonatal brain-MR images demonstrates the efficacy of the proposed segmentation method. Although it is hard to quantify the advantage of the proposed method over other methods without accurate ground-truth manual segmentations, the utility of the proposed method could be more evident in studies involving cortical-thickness measurements and folding-pattern analysis.

\section{Acknowledgments}

This work was funded in part by the USPHS under grants DA-015886, MZ-402069, NS-045839, MH-072576, NINDS K23, and NS052380-03. 


\section{References}

1. van der Knaap, M., Valik, J.: MR imaging of the various stages of normal myelination during the first year of life. Neuroradiology 31(6), 459-470 (1990)

2. Dietrich, R.: Maturation, Myelination, and Dysmyelination. In: Magnetic Resonance Imaging. Mosby pp. 1425-1447 (1999)

3. Barkovich, A.: Magnetic resonance techniques in the assessment of myelin and myelination. J Inherit Metab Dis. 28(3), 311-343 (2005)

4. Prastawa, M., Gilmore, J., Lin, W., Gerig, G.: Automatic segmentation of MR images of the developing newborn brain. Med Image Anal. 9(5), 457-466 (2005)

5. Song, Z., Tustison, N., Avants, B., Gee, J.: Integrated graph cuts for brain mri segmentation. In: Larsen, R., Nielsen, M., Sporring, J. (eds.) MICCAI 2006. LNCS, vol. 4191, pp. 831-838. Springer, Heidelberg (2006)

6. Leemput, K.V., Maes, F., Vandermeulen, D., Seutens, P.: Automated model-based tissue classification of MR images of the brain. IEEE Tr. Med. Imaging 18, 897-908 (1999)

7. Greenspan, H., Ruf, A., Goldberger, J.: Constrained gaussian mixture model framework for automatic segmentation of MR brain images. IEEE Trans. Medical Imaging 25(9), 1233-1245 (2006)

8. Awate, S., Tasdizen, T., Foster, N., Whitaker, R.: Adaptive, nonparametric markov modeling for unsupervised, MRI brain-tissue classification. Medical Image Analysis $10(5), 726-739$ (2006)

9. Awate, S.P., Gee, J.C.: A fuzzy, nonparametric segmentation framework for DTI and MRI analysis. In: Proc. Info. Proc. in Med. Imag (IPMI), (to appear, 2007)

10. Burges, C.: A tutorial on support vector machines for pattern recognition. Data Mining and Knowledge Discovery 2, 121-167 (1998)

11. Boykov, Y., Veksler, O., Zabih, R.: Fast approximate energy minimization via graph cuts. IEEE Trans. Pattern Anal. Machine Intell. 23(11), 1222-1239 (2001)

12. Chow, Y., Geman, S., Wu, L.: Consistent cross-validated density estimation. Annals of Statistics 11(1), 25-38 (1983)

13. Collobert, R., Bengio, S.: Svmtorch: Support vector machines for large-scale regression problems. Journal of machine learning research 1, 143-160 (2001)

14. Platt, J.: Probabilistic outputs for support vector machines and comparison to regularized likelihood methods. In: Advances in Large Margin Classifiers, MIT Press, Cambridge (1999)

15. Weisenfeld, N., Mewes, A., Warfield, S.: Highly accurate segmentation of brain tissue and subcortical gray matter from newborn mri. In: Larsen, R., Nielsen, M., Sporring, J. (eds.) MICCAI 2006. LNCS, vol. 4190, pp. 199-206. Springer, Heidelberg (2006)

16. Avants, B., Gee, J.: Geodesic estimation for large deformation anatomical shape and intensity averaging. Neuroimage Suppl. 1, S139-150 (2004)

17. Likar, B., Viergever, M.A., Pernus, F.: Retrospective correction of MR intensity inhomogeneity by information minimization. IEEE Trans. Med. Imaging 20(12), 1398-1410 (2001)

18. Stark, J.: Adaptive image contrast enhancement using generalizations of histogram equalization. IEEE Trans. Image Processing 9(5), 889-896 (2000) 